\title{
News from other pages
}

Hotez PJ, Aksoy S. Neglected tropical diseases: two years of providing access to innovations for the world's poor... and counting. PLoS Negl Trop Dis. 2009; 3:e494. doi:10.1371/journal.pntd. 0000494.

This open-access journal is the latest addition in the seven currently published open-access PLoS journals (www.plos.org, www.plosntds.org). Currently, the largest numbers of article submissions are from the USA and Brazil, but a large number of submissions come from Asia (India, China, Thailand, Vietnam, and Lao). One article in the current issue of potential interest to Asian readers is "Burkholderia pseudomallei is genetically diverse in agricultural land in northeast Thailand" (by Wuthiekanun V, Limmathurotsakul D, Chantratita N, Feil EJ, Nicholas P. J. Day NPJ, Peacock SJ) and read as follows.

Burkholderia pseudomallei is the cause of melioidosis, a serious human infection most commonly diagnosed in southeast Asia and northern Australia. The organism lives in the soil in a specific geographical distribution and infection results from bacterial inoculation, inhalation or ingestion. The purpose of this study was to define the distribution and genetic diversity of B. pseudomallei in agricultural land where most human infections occur. We performed soil sampling and culture for the presence of $B$. pseudomallei in 100 equally spaced points within a rice paddy in northeast Thailand, and undertook genotyping of primary culture plate colonies from 11 sampling points. We identified seven different genotypes, with relatively limited overlap between different sampling points. Two samples contained more than one B. pseudomallei genotype, in which a numerically dominant genotype coexisted with one or more additional genotypes present as a minority population. We conclude that genetic diversity and structuring of B. pseudomallei exists despite the effects of flooding and the physical and chemical processes associated with farming. These findings inform future efforts to define B. pseudomallei in the environment, and should be considered during the design stage of studies comparing B. pseudomallei isolated from the environment and from patients with invasive disease.

(From Author's Summary, reproduced for Asian Biomedicine under the terms of the Creative Commons Attribution License.) van Thiel P-P AM, de Bie RMA, Eftimov F, Tepaske R, Zaaijer HL, van Doornum GJJ, et al. Fatal human rabies due to duvenhage virus from a bat in Kenya: failure of treatment with coma-induction, ketamine, and antiviral drugs. PloS NTDS Symposium, July 2009

This reports the $3^{\text {rd }}$ case of rabies from the Duvenhage variant, and also has two important teaching points from this reviewer's perspective:

1) The continuing need for lay and professional rabies education (She was not referred by the local authorities for post-exposure treatment)

2) The futility, repeatedly demonstrated, of attempting ketamine-induced-coma treatment, particularly if there is no demonstrable rabies neutralizing antibodies on admission in a patient with clinical signs of rabies.

Scott D, Blizzard L, Fell J., Jones G. Statin therapy, muscle function and falls risk in community-dwelling older adults. Published online 24JUL2009. QJM. doi:10.1093/qjmed/ hcp093. (Accessed 03AUG2009).

This article is a prospective population-based cohort study with a mean follow-up of 2.6 years. Conclusions are as follows. Statin use may exacerbate muscle performance decline and falls risk associated with aging without a concomitant decrease in muscle mass. This effect may be reversed with cessation of statin use.

(Reviewer's comment: Previous studies have not documented statin use as being associated with a reduction in fracture risk (JAMA. 2001; 285:1850). Furthermore, another study on statin use and risk of accidents (Circulation. 2009; 119:2051-7) concluded that statin-adherent patients are systematically more health seeking than those who are not. They also suggested that caution is warranted when interpreting analyses that attribute surprising protective effects to preventive medications. In other words, be careful about confusing association with causation.)

This article was presented by Robert E. Dedmon (E-mail:drbob@aol.com). 